\title{
Some remarks on Thom's transversality theorem
}

\author{
PATRICK BERNARD AND VITO MANDORINO
}

\begin{abstract}
We study Thom's transversality theorem using a point of view, suggested by Gromov, which allows to avoid the use of Sard's theorem and gives finer information on the structure of the set of non-transverse maps.
\end{abstract}

Mathematics Subject Classification (2010): 58B15 (primary); 58A35, 58D20 (secondary).

The smooth image of $\mathbb{R}^{n}$ in $\mathbb{R}^{d}$, for $d>n$ is rectifiable of positive codimension, and therefore it has zero measure. This can be seen as the easy case of Sard's Theorem, which states that the set of critical values of a smooth map has zero measure. The most common proofs of the Thom transversality theorem rely on the general form of Sard's theorem. Our goal in the present paper is to develop an approach, suggested by Gromov, in [5, page 33], and used in [8, Section 2.3] to this theorem using only the easy case of Sard's Theorem, which leads to a stronger form of Thom's theorem. The key technical tool in this approach is our Conjecture 3.1 below. The main novelty in the present paper is the proof of several cases of that conjecture, Theorems 3.2 and 3.3 in Section 3. These special cases are enough to derive new variants of the Thom transversality theorem, stated as Theorem 1.4 in the introduction. We work in a context closely inspired from [1], and we use a notion of rectifiable sets in Banach spaces coming from [14] and [4], as recalled in Section 2.

\section{Introduction}

It is well-known that "most" functions are Morse, which means that their critical points are non-degenerate. Discussing this claim with some details will be an occasion to introduce and motivate the present work. Let us fix some integer $r \geqslant 2$ and a dimension $n$. Let $B^{n}$ be the open unit ball in $\mathbb{R}^{n}$, and $\bar{B}^{n}$ be the closed unit ball. We denote by $C^{r}\left(\bar{B}^{n}, \mathbb{R}\right)$ the space of functions which are $C^{r}$ on $B^{n}$, and whose differentials up to order $r$ extend by continuity to the closed ball $\bar{B}^{n}$. We endow this space with the norm given by the sum of the supremums of the differentials of order less than $r$. It is then a separable Banach space. Let $F$ be an affine subspace 
of $C^{r}\left(\bar{B}^{n}, \mathbb{R}\right)$. In most cases $F$ will just be the whole space $C^{r}\left(\bar{B}^{n}, \mathbb{R}\right)$, but it is sometimes useful to consider finite-dimensional spaces $F$. The map

$$
\begin{aligned}
e_{1}: B^{n} \times F & \longrightarrow \mathbb{R}^{n} \\
(x, f) & \longmapsto d f(x)
\end{aligned}
$$

is $C^{r-1}$, and, when $F=C^{r}\left(\bar{B}^{n}, \mathbb{R}\right)$ it is a submersion, see [1, Theorem 10.4]. Recall that a $C^{1}$ map is a submersion if and only if its differential at each point is onto with a split kernel. Then, it is locally equivalent (by left and right composition by $C^{1}$ maps) to a projection. We will always assume that $F$ is chosen such that $e_{1}$ is a submersion (or at least that it is transverse to $\{0\}$ ). Let us then denote by $\Sigma_{1}$ the manifold $e_{1}^{-1}(0)$, and consider the restriction $\pi_{\mid \Sigma_{1}}$ to $\Sigma_{1}$ of the projection on the second factor. This map is $C^{1}$, and it is Fredholm of index 0. Moreover a map $f_{0} \in F$ is Morse (on $B^{n}$ ) if and only if it is a regular value of $\pi_{\mid \Sigma_{1}}$, which means that the differential of this map is onto at each point $\left(x, f_{0}\right)$ of $\Sigma_{1}$. These claims are proved in [1], Section 19, the argument is recalled in Section 2.2 for the convenience of the reader, see Proposition 2.2. We have proved that the set $N \subset F$ of non-Morse functions can be written as

$$
N=C V\left(\pi_{\mid \Sigma_{1}}\right),
$$

where $C V$ denotes the set of critical values. By the theorem of Sard and Smale (see Section 2), this set is Baire-meager, and it has zero measure in $F$, in a sense that will be made precise in Section 2.

Let us now present, for $r \geqslant 3$, a slightly different approach which has the advantage of avoiding the use of the Theorem of Sard and Smale. Denoting by $S^{n}$ the set of symmetric $d \times d$ matrices, we start with the evaluation map

$$
\begin{aligned}
e_{2}: B^{n} \times F & \longrightarrow \mathbb{R}^{n} \times S^{n} \\
(x, f) & \longmapsto\left(d f(x), d^{2} f(x)\right)
\end{aligned}
$$

which is $C^{r-2}$ and, when $r \geqslant 3$ and $F=C^{r}\left(\bar{B}^{n}, \mathbb{R}\right)$, is a submersion. Let us denote by $\tilde{A} \subset \mathbb{R}^{n} \times S^{n}$ the subset of points $(0, H)$, with $H$ singular. Note that $\tilde{A}$ is an algebraic submanifold of codimension $n+1$ in $\mathbb{R}^{n} \times S^{n}$, hence a finite union of smooth submanifolds of codimension at least $n+1$. The set $N \subset F$ of non-Morse functions can be written

$$
N=\pi\left(\Sigma_{2}\right), \quad \Sigma_{2}=e_{2}^{-1}(\tilde{A}) .
$$

It is best here to first consider that $F$ is finite-dimensional (but that $e_{2}$ is still a submersion). Then, $\Sigma_{2}$ is a finite union of manifolds of dimension less than $\operatorname{dim} F$. This implies that $N=\pi\left(\Sigma_{2}\right)$ is rectifiable of dimension less than $\operatorname{dim} F$, or in other words it is rectifiable of positive codimension in $F$. This implies that $N$ has zero measure, but is a much more precise information, which was obtained without the use of Sard Theorem. This reasoning can be extended to the case where $F$ is not 
finite-dimensional with the help of an appropriate notion of rectifiable sets recalled in Section 2. More precisely, we know that $\Sigma_{2}$ is a finite union of manifolds of codimension at least $n+1$. Since $\pi$ is obviously Fredholm of index $n$, we conclude by Proposition 2.6 that $\pi\left(\Sigma_{2}\right)$ is rectifiable of codimension 1 . We obtain:

Theorem 1.1. Let $N \subset C^{r}\left(\bar{B}^{n}, \mathbb{R}\right)$ be the set of functions which are not Morse on $B^{n}$.

- If $r \geqslant 2$ then $N$ is a countable union of closed sets with empty interior, it has zero measure (in the sense of Haar or Aronszajn).

- If $r \geqslant 3$ then $N$ is rectifiable of positive codimension.

The concepts of sets of zero measure (Haar-null or Aronszajn-null sets) in separable Banach spaces used in this statement are recalled in Section 2, together with the concept of rectifiable set of positive codimension. Each point in the statement is the result of one of the strategies of proof exposed above; notice that none of these statements contains the other.

As a second illustration, we consider a smooth manifold $A \subset \mathbb{R}^{m}$ and describe the set $N A \subset F=C^{r}\left(\bar{B}^{n}, \mathbb{R}^{m}\right)$ of maps which are not transverse to $A$ on $B^{n}$. We consider the evaluation map

$$
\begin{aligned}
E_{0}: B^{n} \times F & \longrightarrow \mathbb{R}^{m} \\
(x, f) & \longmapsto f(x) .
\end{aligned}
$$

This map is $C^{r}$ and, for $r \geqslant 1$, it is a submersion. We then have

$$
N A=C V\left(\pi_{\mid \Sigma_{0}}\right), \quad \Sigma_{0}=E_{0}^{-1}(A),
$$

and $\pi_{\mid \Sigma_{0}}$ is $C^{r}$ and Fredholm of index $i=n-c$, where $c$ is the codimension of $A$, as follows from Proposition 2.2. If $r \geqslant n-c+1$, we can apply the theorem of Sard and Smale (see Section 2), and obtain that this set is Baire-meager, and has zero measure in $F$, in a sense that will be made precise in Section 2 . When $c>n$, we also conclude that $N A=\pi\left(\Sigma_{0}\right)$ is rectifiable of positive codimension.

The second approach, which is useful for $c \leqslant n$, consists in using the evaluation map

$$
\begin{gathered}
E_{1}: B^{n} \times F \longrightarrow \mathbb{R}^{m} \times L\left(\mathbb{R}^{n}, \mathbb{R}^{m}\right) \\
(x, f) \longmapsto\left(f(x), d f_{x}\right) .
\end{gathered}
$$

This map is $C^{r-1}$, and, for $r \geqslant 2$, it is a submersion. Let us denote by $\tilde{A}$ the set of pairs $(y, l) \in \mathbb{R}^{m} \times L\left(\mathbb{R}^{n}, \mathbb{R}^{m}\right)$ such that $y \in A$ and $l\left(\mathbb{R}^{n}\right)+T_{y} A \subsetneq \mathbb{R}^{m}$. We then have

$$
N A=\pi\left(\tilde{\Sigma}_{1}\right), \quad \tilde{\Sigma}_{1}=E_{1}^{-1}(\tilde{A}) .
$$


We conclude as above that $N A$ is rectifiable of positive codimension in view of the following:

Lemma 1.2. The set $\tilde{A}$ is a countable union of smooth manifolds of codimension more than $n$.

Proof. Locally, there exists a submersion $F: \mathbb{R}^{m} \longrightarrow \mathbb{R}^{c}$ such that $A=F^{-1}(0)$. Then, the set $\tilde{A}$ is the preimage by the local submersion

$$
\mathbb{R}^{m} \times L\left(\mathbb{R}^{n}, \mathbb{R}^{m}\right) \ni(y, l) \longmapsto\left(F(y), d F_{y} \circ l\right) \in \mathbb{R}^{c} \times L\left(\mathbb{R}^{n}, \mathbb{R}^{c}\right)
$$

of the set $B:=\{0\} \times L_{S}\left(\mathbb{R}^{n}, \mathbb{R}^{c}\right)$ where $L_{S}$ is the set of singular linear maps from $\mathbb{R}^{n}$ to $\mathbb{R}^{c}$ (maps of rank less than $c$ ). It is well-known that $L_{S}\left(\mathbb{R}^{n}, \mathbb{R}^{c}\right.$ ) is an analytic submanifold of codimension $n-c+1$ in $L\left(\mathbb{R}^{n}, \mathbb{R}^{c}\right)$, hence $B$ is an analytic submanifold of codimension $n+1$. As a consequence, $B$ is a finite union of smooth submanifolds of codimension at least $n+1$, hence so is $\tilde{A}$.

As a conclusion, we obtain:

Theorem 1.3. Let $A$ be a smooth submanifold of $\mathbb{R}^{m}$ of codimension $c$.

- For $r \geqslant n-c+1$, The set $N A$ is Baire meager and Aronzajn-null (hence Haar-null) in $C^{r}\left(\bar{B}^{n}, \mathbb{R}^{m}\right)$.

- For $r \geqslant 2$, the set $N A$ is rectifiable of positive codimension in $C^{r}\left(\bar{B}^{n}, \mathbb{R}^{m}\right)$, it is thus Baire meager and Aronszajn-null.

It is worth observing that the second statement contains the first one, except for the case where $c=n$ and $r=1$. Our goal in the present paper is to develop an analogue of the second strategy presented on the examples above to prove the Thom transversality theorem in the space of jets. This idea was suggested by Gromov, in [5, page 33], and used in [8, Section 2.3.], where it is reduced to an appropriate generalization of Lemma 1.2 above. This lemma, which is stated there without proof, is our Conjecture 1.5. The main novelty in the present paper consists in giving a full proof of this conjecture in the analytic case. We also explain that this strategy, as in the examples above, leads to a more precise statement of the Thom transversality theorem than the usual proof based on the Theorem of Sard:

Theorem 1.4. Let $A$ be a smooth submanifold of $J^{p}\left(\bar{B}^{n}, Y\right)$ of codimension $c$, where $Y$ is a finite-dimensional separable manifold. For $r \geq p+1$, let $N A \subset$ $C^{r}\left(\bar{B}^{n}, Y\right)$ be the set of maps whose p-jet is not transverse to $A$.

- If $c \geqslant n+1$ and $r \geqslant p+1$ then the set $N A$ is rectifiable of codimension $c-n$ in $C^{r}\left(\bar{B}^{n}, Y\right)$; it is thus Baire meager and Aronszajn-null.

- If $c \leqslant n$ and $r \geqslant p+1+n-c$, then the set $N A$ is Baire meager and Aronzajnnull (hence Haar-null) in $C^{r}\left(\bar{B}^{n}, Y\right)$.

- If $c \leqslant n$ and $r \geqslant p+2$ and $A$ is analytic, then the set $N A$ is rectifiable of positive codimension in $C^{r}\left(\bar{B}^{n}, Y\right)$; it is thus Baire meager and Aronszajn-null. 
Proof. For completeness, we first quickly recall the usual proof of the Thom Transversality Theorem, as given in [1], which yields the second point of the theorem. We consider the evaluation map (with $F=C^{r}\left(\bar{B}^{n}, Y\right)$ ):

$$
\begin{aligned}
E_{p}: B^{n} \times F & \longrightarrow J^{p}\left(\bar{B}^{n}, Y\right) \\
(x, f) & \longmapsto j_{x}^{p} f .
\end{aligned}
$$

This map is $C^{r-p}$, and it is a submersion, see [1, Theorem 10.4]. It follows from Proposition 2.2 below that

$$
N A=C V\left(\pi_{\mid \Sigma}\right), \quad \Sigma=E_{p}^{-1}(A)
$$

Moreover, the map $\pi_{\mid \Sigma}$ is Fredholm of index $i=n-c$. We conclude from the Theorem of Sard and Smale (Theorem 2.12 below) that $N A$ has zero measure and is Baire meager. If, in addition, the codimension of $A$ is larger than $n$, then so is the codimension of $\Sigma$, and we can conclude directly by the "Easy Part" of the theorem of Sard and Smale that $N A$ is rectifiable of positive codimension. For the case where $c \leqslant n$, we obtain the proof of the last point of the theorem by considering the evaluation map

$$
\begin{gathered}
E_{p+1}: B^{n} \times F \longrightarrow J^{p+1}\left(\bar{B}^{n}, Y\right) \\
(x, f) \longmapsto j_{x}^{p+1} f,
\end{gathered}
$$

which is a $C^{r-p-1}$ submersion, and the set

$$
\tilde{A} \stackrel{\text { def }}{=}\left\{j_{x}^{p+1} f \in J^{p+1}\left(\bar{B}^{n}, Y\right): j^{p} f \text { is not transverse to } A \text { at } x\right\} \subseteq J^{p+1}\left(\bar{B}^{n}, Y\right) \text {. }
$$

By definition, we have

$$
N A=\pi(\tilde{\Sigma}), \quad \tilde{\Sigma}=E_{p+1}^{-1}(\tilde{A}) .
$$

The last point of Theorem 1.4, without the additional restriction on $A$, would be a consequence of the following conjecture. The cases of the conjecture that we will be able to prove, Theorem 3.3 in Section 3, imply Theorem 1.4.

Conjecture 1.5. If $A$ is a smooth submanifold of $J^{p}\left(\bar{B}^{n}, Y\right)$ of codimension $c \leqslant n$, then $\tilde{A}$ is a countable union of smooth submanifolds of codimension more than $n$ in $J^{p+1}\left(\bar{B}^{n}, Y\right)$.

In view of Proposition 2.10, it would even be enough for our applications to prove that $\tilde{A}$ is rectifiable of codimension $n+1$ (in the sense of Section 2.3). We come back to this conjecture in Section 3, where we obtain some special cases, see Theorem 3.2 and 3.3, which are sufficient to imply the third point of Theorem 1.4. In Section 2, we recall several mathematical notions which have been used in this introduction. 


\section{Small sets, rectifiable sets, and the theorem of Sard and Smale}

\subsection{Some notions of small sets}

Let $F$ be a separable Banach space. We define below three translation invariant $\sigma$-ideals of subsets of $F$. A $\sigma$-ideal is a family $\mathcal{F}$ of subsets of $F$ such that

$$
\begin{aligned}
A & \in \mathcal{F}, A^{\prime} \subset A \Rightarrow A^{\prime} \in \mathcal{F}, \\
\forall n \in \mathbb{N}, A_{n} \in \mathcal{F} & \Rightarrow \cup_{n \in \mathbb{N}} A_{n} \in \mathcal{F} .
\end{aligned}
$$

A subset $A \subset F$ is called Baire-meager if it is contained in a countable union of closed sets with empty interior. The Baire Theorem states that a Baire-meager subset of a Banach space has empty interior.

A subset $A \subset F$ is called Haar-null if there exists a Borel probability measure $\mu$ on $F$ such that $\mu(A+f)=0$ for all $f \in F$. The equality $\mu(A+f)=0$ means that the set $A+f$ is contained in a Borel set $\tilde{A}_{f}$ such that $\mu\left(\tilde{A}_{f}\right)=0$. A countable union of Haar-null sets is Haar-null, see [3,6] and [11] for the non-separable case.

A subset $A \subset F$ is called Aronszajn-null if, for each sequence $f_{n}$ generating a dense subset of $F$, there exists a sequence $A_{n}$ of Borel subsets of $F$ such that $A \subset \cup_{n} A_{n}$ and such that, for each $f \in F$ and for each $n$, the set

$$
\left\{x \in \mathbb{R}: f+x f_{n} \in A_{n}\right\} \subset \mathbb{R}
$$

has zero Lebesque measure. A countable union of Aronszajn-null sets is Aronszajnnull, and each Aronszajn-null set is Haar null, see [2,3].

The notion of probe allows a simple criterion for proving that a Borel set $A$ is Haar or Aronszajn null. A probe for $A$ is a finite-dimensional vector space $E \subset F$ such that $(A+f) \cap E$ has Lebesgue measure zero in $E$ for each $f \in F$. It is easy to see that $A$ is Haar null if there exists a probe for $A$. In the sense of Aronszajn, we have (see [14, Proposition 4.3]):

Lemma 2.1. Let $A \subset F$ be a Borel set. If the set of probes for A contains a non-empty open set in the space of finite dimensional subspaces of $F$, then $A$ is Aronszajn null.

Proof. Let $f_{n}$ be a sequence of points of $F$ generating a dense subspace. Under the hypothesis of the lemma, there exists $N \in \mathbb{N}$ and a probe $E$ such that $E \subset$ $\operatorname{Vect}\left(f_{n}, n \leqslant N\right)$. Then, the space $F_{N}:=\operatorname{Vect}\left(f_{n}, n \leqslant N\right)$ is itself a probe for $A$. By standard arguments, (see [3, Proposition 6.29] or [14]), we conclude that $A=\cup_{n \leqslant N} A_{n}$, where each $A_{n}$ is a Borel set such that the set

$$
\left\{x \in \mathbb{R}: f+x f_{n} \in A_{n}\right\} \subset \mathbb{R}
$$

has zero measure for each $f \in F$. Since this holds for each sequence $f_{n}$ with dense range, we conclude that $A$ is Aronszajn null. 
If $X$ is a separable manifold modeled on a separable Banach space $F_{X}$, we also have notions of Baire meager, Haar null and Aronszajn null subsets of $X$. We say that $A \subset X$ is Baire meager, Haar null or Aronszajn null if, for each $C^{1}$ chart $\varphi: B_{X} \longrightarrow X$, the set $\varphi^{-1}(A)$ is Baire meager, Haar null or Aronszajn null, where $B_{X}$ is the open unit ball in $F_{X}$. Baire meager sets can also be defined directly as subsets of countable unions of closed sets with empty interior in the Baire topological space $X$.

The situation is slightly more problematic with Haar-null or Aronszajn-null sets, because these $\sigma$-ideals are not invariant under $C^{1}$ diffeomorphisms. As a consequence, being Haar null or Aronszajn null in the Banach space $F_{X}$ seen as a Banach Manifold is a stronger property than being Haar null or Aronszajn null in $F_{X}$ seen as a Banach space. This ambiguity in terminology should not cause problems in the sequel. Many other notions of sets of zero measure in nonlinear spaces have been introduced, see for example the survey [10]; see also [13] for some applications in PDE.

\subsection{Fredholm maps}

Given Banach spaces $F$ and $B$, a continuous linear map $L: F \longrightarrow B$ is called Fredholm if its kernel is finite-dimensional and if its range is closed and has finite codimension. We say that $L$ is a Fredholm linear map of type $(k, l)$ if $k$ is the dimension of the kernel of $L$ and $l$ is the codimension of its range. The index of $L$ is the integer $k-l$. Recall that the set of Fredholm linear maps is open in the space of continuous linear maps (for the norm topology), and that the index is locally constant, although the integers $k$ and $l$ are not. They are lower semi-continuous. When $F$ and $B$ have finite dimension $n$ and $m$, then the index of all linear maps is $i=n-m$.

The following essentially comes from [1, Section 19]:

Proposition 2.2. Let $F, X$ be Banach spaces such that $X$ has finite dimension $n$. Let $l: F \times X \longrightarrow \mathbb{R}^{c}$ be a surjective continuous linear map, let $K$ be the kernel of $l$, and let $k$ be the restriction to $K$ of the projection $(f, x) \longmapsto f$. Then $k$ is Fredholm of index $n-c$. Moreover, it is onto if and only if the restriction $l_{0}$ of $l$ to $\{0\} \times X$ is onto.

Proof. Let us denote by $X_{0}$ the space $\{0\} \times X$, by $F_{0}$ the space $F \times\{0\}$, and by $K_{0}$ the intersection $K \cap X_{0}$. To prove that the continuous linear map $k$ is Fredholm, we write

$$
F \times X=K_{1} \oplus K_{0} \oplus X_{1} \oplus F_{1}
$$

where

- $F_{1} \subset F_{0}$ and $F_{1} \oplus\left(K+X_{0}\right)=F \times X$. Such a space exists because $K+X_{0}$ has finite codimension, and because $F_{0}+K+X_{0}=F \times X$,

- $K_{1} \oplus K_{0}=K$,

- $X_{1} \oplus K_{0}=X_{0}$. 
Denoting by $\pi$ the projection on the first factor, we see that the restriction of $\pi$ to $K_{1} \oplus F_{1}$ is an isomorphism onto $F$. This implies that the map $k$ is conjugated to linear map

$$
\begin{gathered}
K_{1} \oplus K_{0} \longrightarrow K_{1} \oplus F_{1} \\
\kappa_{1}+\kappa_{0} \longmapsto \kappa_{1}+0,
\end{gathered}
$$

which is Fredholm of index $i=\operatorname{dim} K_{0}-\operatorname{dim} F_{1}$. We obtain that

$$
i=\left(\operatorname{dim} K_{0}+\operatorname{dim} X_{1}\right)-\left(\operatorname{dim} X_{1}+\operatorname{dim} F_{1}\right)=n-c .
$$

The linear Fredholm map $k$ is onto if and only if its kernel $K_{0}$ has dimension $k=$ $n-c$. On the other hand, the space $K_{0}$ is also the kernel of $l_{0}$, hence it has dimension $n-c$ if and only if the $l_{0}$ is onto $\left(X_{0}\right.$ has dimension $n$ ). We have proved the second part of the statement.

We now recall a standard lemma of differential calculus (this lemma can be applied in particular to Fredholm maps):

Lemma 2.3. Let $f: X \longrightarrow Y$ be a $C^{1}$ map and $x_{0}$ be a point such that $d f_{x_{0}}$ has a closed and split range $I \subset F_{Y}$ and a split kernel $K \subset F_{X}$. Let $G$ be a supplement of $I$ in $F_{Y}$. Then, for each local diffeomorphism $\phi:\left(Y, f\left(x_{0}\right)\right) \longrightarrow(I \times G, 0)$ there exists a local diffeomorphism $\varphi:(I \times K, 0) \longrightarrow\left(X, x_{0}\right)$ such that

$$
\phi \circ f \circ \varphi\left(x_{i}, x_{k}\right)=\left(x_{i}, \psi\left(x_{i}, x_{k}\right)\right)
$$

for some $C^{1}$ local map $\psi: I \times K \longrightarrow G$.

Proof. Let $E$ be a supplement of $K$ in $F_{X}$. By considering first an arbitrary local chart $\tilde{\varphi}:(E \times K, 0) \longrightarrow\left(X, x_{0}\right)$, we write

$$
\phi \circ f \circ \tilde{\varphi}:\left(x_{e}, x_{k}\right) \longmapsto\left(f_{i}\left(x_{e}, x_{k}\right), f_{g}\left(x_{e}, x_{k}\right)\right) \text {. }
$$

It follows from the definition of $G$ and $I$ that $\partial_{x_{e}} f_{i}$ is an isomorphism, hence the mapping

$$
\left(x_{e}, x_{k}\right) \longmapsto\left(f_{i}\left(x_{e}, x_{k}\right), x_{k}\right)
$$

is a local diffeomorphism between $\left(B_{X}, 0\right)$ and $(I \times K, 0)$. Denoting by $\hat{\varphi}\left(x_{i}, x_{k}\right)=$ $\left(\hat{\varphi}_{e}\left(x_{i}, x_{k}\right), x_{k}\right)$ its inverse, we see that

$$
\phi \circ f \circ \tilde{\varphi} \circ \hat{\varphi}\left(x_{i}, x_{k}\right)=\left(x_{i}, \psi\left(x_{i}, x_{k}\right)\right)
$$

with $\psi\left(x_{i}, x_{k}\right)=f_{g}\left(\hat{\varphi}_{e}\left(x_{i}, x_{k}\right), x_{k}\right)$.

We also recall the constant rank (or rather constant corank) theorem. 
Lemma 2.4. Let $f: X \longrightarrow Y$ be a $C^{1}$ map. Assume that there exists an integer $c$ such that, for each $x \in X, d f_{x}$ has a closed range $I \subset F_{Y}$ of codimension $c$ and a split kernel $K \subset F_{X}$. Let $G$ be a supplement of $I$ in $F_{Y}$. Then, near each point $x_{0} \in X$ there exists a local diffeomorphism $\phi:\left(Y, f\left(x_{0}\right)\right) \longrightarrow(I \times G, 0)$ and a local diffeomorphism $\varphi:(I \times K, 0) \longrightarrow\left(X, x_{0}\right)$ such that

$$
\phi \circ f \circ \varphi\left(x_{i}, x_{k}\right)=\left(x_{i}, 0\right) .
$$

Proof. We first apply Lemma 2.3 and find charts $\tilde{\phi}$ and $\varphi$ such that $\tilde{\phi} \circ f \circ \varphi\left(x_{i}, x_{k}\right)=$ $\left(x_{i}, \psi\left(x_{i}, x_{k}\right)\right)$. The differential of this map has corank $c$ (which is the dimension of $G$ ) if and only if $\partial_{x_{k}} \psi=0$. We conclude that $\psi$ does not depend on $x_{k}$. We now set $\hat{\phi}\left(x_{i}, x_{g}\right)=\left(x_{i}, x_{g}-\psi\left(x_{i}\right)\right)$, and observe that $\hat{\phi} \circ \tilde{\phi} \circ f \circ \varphi\left(x_{i}, x_{k}\right)=\left(x_{i}, 0\right)$.

\subsection{Rectifiable sets in Banach manifolds}

We use here the definition of rectifiable sets of finite codimension given in [4], which extrapolates on [14]. Our terminology, however, differs from that of [4] : we call rectifiable here what we called countably rectifiable there.

The subset $A$ in the Banach space $F$ is a Lipschitz graph of codimension $\mathbf{d}$ if there exists a splitting $F=E \oplus G$, with $\operatorname{dim} G=d$ and a Lipschitz map $g: E \longrightarrow$ $G$ such that

$$
A \subset\{x+g(x), x \in E\} .
$$

Let $X$ be a separable manifold modeled on the separable Banach space $F_{X}$. A subset $A \subset X$ is rectifiable of codimension $\mathbf{d}$ if it is a countable union $A=\cup_{n} \varphi_{n}\left(A_{n}\right)$ where

- $\varphi_{n}: U_{n} \longrightarrow X$ is a Fredholm map ${ }^{1}$ of index $i_{n}$ defined on an open subset $U_{n}$ in a separable Banach space $F_{n}$,

- $A_{n} \subset U_{n}$ is a Lipschitz graph of codimension $d+i_{n}$ in $F_{n}$.

Note that, by definition, if $A \subset X$ is rectifiable of codimension $d$ then it is rectifiable of codimension $d^{\prime}$ for all $0 \leq d^{\prime} \leq d$. The following properties are proved in [14] or [4].

Proposition 2.5. A rectifiable set of positive codimension is Baire meager. More precisely, it is contained in a countable union of closed sets of positive codimension. It is also Aronszanjn null, hence Haar null.

Proposition 2.6. Let $X$ and $Y$ be separable Banach manifolds, and let $f: X \longrightarrow$ $Y$ be $C^{1}$ Fredholm of index $i$, and let $A \subset X$ be rectifiable of codimension $d \geqslant i+1$, then the direct image $f(A)$ is rectifiable of codimension $d-i$.

${ }^{1}$ A Fredholm map of index $i$ between separable Banach manifolds is a $C^{1}$ map such that the differential is Fredholm of index $i$ at every point (recall that the index is locally constant). 
The following property is almost taken from [4]:

Proposition 2.7. Let $X$ and $Y$ be separable Banach manifolds, let $A \subset Y$ be rectifiable of codimension $d$ and let $f: X \longrightarrow Y$ be a $C^{1}$ map such that, at each point of $f^{-1}(A)$, the differential $d f$ has the following properties with some integer $k \leqslant d-1$ :

- It has a split kernel,

- It has a closed image of codimension at most $k$.

Then, $f^{-1}(A)$ is rectifiable of codimension $d-k$. In particular, if $f$ is a submersion, then $f^{-1}(A)$ is rectifiable of codimension $d$.

Proof. In view of Lemma 2.3, it is enough to prove the statement for maps of the form $\left(x_{i}, x_{k}\right) \longmapsto\left(x_{i}, \psi\left(x_{i}, x_{k}\right)\right)$, where $\left(x_{i}, x_{k}\right) \in I \times K$, and $\psi$ takes value in $G$, a supplement of $I$ in $B_{Y}$ (hence $\operatorname{dim} G \leqslant k$ ). We also consider that $A \subset I \times G$ is rectifiable of codimension $d$. Then the projection $A_{I}$ of $A$ on $I$ is rectifiable of codimension $d-k$ in $I$. In view of the special form of the map we consider, the preimage of $A$ is contained in $A_{I} \times K$, which is rectifiable of codimension $d-k$ in $I \times K$ because $A_{I}$ is rectifiable of codimension $d-k$ in $I$.

We express the following results in the context of Banach spaces to avoid some technical complications. If $F$ is a separable Banach space, then we define the separable Banach spaces $C^{p}\left(\bar{B}^{n}, F\right)$ as in the introduction. The following result was proved in [4]:

Proposition 2.8. If $A \subset C^{p}\left(\bar{B}^{n}, F\right)$ is rectifiable of codimension $d$, and $p^{\prime} \geqslant p$, then $A \cap C^{p^{\prime}}\left(\bar{B}^{n}, F\right)$ is rectifiable of codimension $d$ in $C^{p^{\prime}}\left(\bar{B}^{n}, F\right)$.

This results allows to define sets of positive codimension in the Frechet space $C^{\infty}\left(\bar{B}^{n}, F\right)$, see [1]. The following result makes precise the simple fact that "most" $n$-parameter families avoid sets of codimension $d$ when $d>n$.

Proposition 2.9. Let $F$ be a separable Banach space, and $A \subset F$ a rectifiable set of codimension $d$. For $n<d$, The set $\mathcal{A} \subset C^{1}\left(\bar{B}^{n}, F\right)$ of maps $f$ such that $f\left(B^{n}\right) \cap A \neq \emptyset$ is rectifiable of codimension $d-n$.

Proof. This is just a variant of the methods of proof used in the introduction. We consider the evaluation map

$$
E_{0}: B^{n} \times C^{p}\left(\bar{B}^{n}, F\right) \longrightarrow F
$$

which is a $C^{1}$ submersion. We conclude from Proposition 2.7 that $E_{0}^{-1}(A)$ is rectifiable of codimension $d$ in $B^{n} \times C^{p}\left(\bar{B}^{n}, F\right)$. The set $\mathcal{A}$, which is the projection of $E_{0}^{-1}(A)$ on the second factor, is thus rectifiable of codimension $d-n$. 
The "easy case" of the transversality theorem also has a natural analogue in terms of rectifiable sets:

Proposition 2.10. Let $F$ be a separable Banach space, and $A \subset J^{p}\left(\bar{B}^{n}, F\right)$ a rectifiable set of codimension $d$. For $n<d$, The set $\mathcal{A} \subset C^{p+1}\left(\bar{B}^{n}, F\right)$ of maps $f$ such that $j^{p} f\left(B^{n}\right) \cap A \neq \emptyset$ is rectifiable of codimension $d-n$.

Proof. It is the same as above, using the evaluation map $E_{p}:(x, f) \longmapsto j_{x}^{p} f$.

\subsection{The Theorem of Sard and Smale}

Theorem 2.11. Let $X$ be a smooth (separable) manifold of dimension $n, Y$ be a smooth manifold of dimension $m$, and let $f: X \longrightarrow Y$ be a $C^{r}$ map. If $r \geqslant$ $1+(n-m)$, and $m \leqslant n$ then the set $C V(f)$ of critical values of $f$ has zero measure in $Y$.

The theorem also holds in the case where $r \geqslant 1$ and $n \leqslant m-1$, which is sometimes called the easy case of Sard's theorem. In this case, however, the set $C V(f)$ is just the image $f(X)$, which is rectifiable of dimension $n$ in $Y$. Since $n<m$, this implies the result, but is a much finer information. The theorem of Sard was extended by Smale to the infinite-dimensional case. We give below a more precise statement:

Theorem 2.12. Let $X$ and $Y$ be separable smooth manifolds modelled on separable Banach spaces, and let $f: X \longrightarrow Y$ be a $C^{r}$ Fredholm map of index $i$.

- If $i \geqslant 0$ and $r \geqslant 1+i$ then the set $C V(f)$ of critical values of $f$ is Aronszajn null (hence Haar null) and Baire meager in $Y$,

- If $i<0$ and $r \geqslant 1$ then the set $f(X)=C V(f)$ is rectifiable of codimension $-i$ in $Y$ (it is thus Aronszajn-null and Baire meager).

Proof. The second part of the statement (the "easy case"), is a special case of Proposition 2.6. Let us focus on the first part. Let $P$ be the set of critical points of $f$, so that $C V(f)=f(P)$.

We claim that each point $x_{0}$ of $P$ has a closed neighborhood $\tilde{P}$ in $P$ such that $f(\tilde{P})$ is closed and Aronszajn-null. Since $P$ is a separable metric space, it has the Lindelöf property, and it can be covered by countably many such local sets $\tilde{P}$. As a consequence, the claim implies the statement.

Since the claim is local, we identify $X$ with its Banach model $B_{X}$ and $x_{0}$ with 0 , and similarly $\left(Y, f\left(x_{0}\right)\right)$ with $\left(B_{Y}, 0\right)$. Let $I$ be the range of $d f_{0}$, and let $G$ be a supplement of $I$ in $F_{Y}$, note that $G$ has finite dimension $l$. By Lemma 2.3, there exists a local diffeomorphism $\varphi:(I \times K, 0) \longrightarrow\left(B_{X}, 0\right)$ such that

$$
f \circ \varphi\left(x_{i}, x_{k}\right)=x_{i}+\psi\left(x_{i}, x_{k}\right),
$$

where $\psi: I \times K \longrightarrow G$ is $C^{r}$. We conclude that

$$
\phi(f(\tilde{P})) \subset C V(\phi \circ f \circ \varphi) \subset \bigcup_{x_{i} \in I}\left(x_{i}+C V\left(\psi_{x_{i}}\right)\right)
$$


where $\psi_{x_{i}}: K \longrightarrow G$ is the map $x_{k} \longmapsto \psi\left(x_{i}, x_{k}\right)$. In view of the finitedimensional Sard theorem (applied to $\psi_{x_{i}}$ ), we conclude that $G$ is a probe for $\phi(f(\tilde{P}))$. Since it admits a probe, this set is Haar-null. Moreover, since the set of supplements of the $I$ is open in the space of $l$-dimensional subspaces of $F_{Y}$, we conclude from Lemma 2.1 that $\phi(f(\tilde{P}))$ is Aronszajn-null. Since this holds for each local chart $\phi$, we have proved that $f(\tilde{P})$ is Aronszajn-null in $Y$.

Finally, let us prove that $f(\tilde{P})$ is closed, or equivalently that $\phi(f(\tilde{P}))$ is closed, provided $\tilde{P}$ is chosen bounded and closed in $B_{X}$. Let $x^{n}$ be a sequence in $\tilde{P}$, such that $f\left(x^{n}\right)$ has a limit $y^{\infty}$, we have to prove that $y^{\infty} \subset f(\tilde{P})$. Let us denote by $\left(x_{i}^{n}, x_{k}^{n}\right)$ the sequence $\varphi^{-1}\left(x^{n}\right)$. Since $K$ is finite-dimensional and $x^{n}$ is bounded, we can assume by taking a subsequence that $x_{k}^{n}$ has a limit $x_{k}^{\infty}$. On the other hand, since $f\left(x^{n}\right) \longrightarrow y^{\infty}$, we conclude that

$$
\left(x_{i}^{n}, \psi\left(x_{i}^{n}, x_{k}^{n}\right)\right)=\phi \circ f \circ \varphi\left(x_{i}^{n}, x_{k}^{n}\right)=\phi \circ f\left(x^{n}\right) \longrightarrow \phi\left(y^{\infty}\right),
$$

hence $x_{i}^{n}$ has a limit $x_{i}^{\infty}$ (which is the first component of $\phi\left(y^{\infty}\right)$ ). The sequence $\left(x_{i}^{n}, x_{k}^{n}\right)$ is thus convergent, hence so is $x_{n}=\varphi\left(x_{i}^{n}, x_{k}^{n}\right)$. Since $\tilde{P}$ is closed, the limit $x^{\infty}$ belongs to $\tilde{P}$, and $y^{\infty}=f\left(x^{\infty}\right)$.

\section{Some cases of the conjecture}

In this section we consider two finite-dimensional smooth manifolds $X$ and $Y$. For $p \in \mathbb{N}$, we denote by $J^{p}(X, Y)$ the space of $p$-jets of functions $X \longrightarrow Y$. See for example $[9,12]$ for some details on jet bundles. Note that $J^{0}(X, Y)=X \times Y$, and it will also be convenient to consider that $J^{-1}(X, Y)=X$. For $p \leqslant p^{\prime}$ we have a natural projection

$$
\pi_{p}^{p^{\prime}}: J^{p^{\prime}}(X, Y) \longrightarrow J^{p}(X, Y)
$$

When $p=-1$, this is just the source map $j_{x}^{p^{\prime}} f \longmapsto x$. When $p \in \mathbb{N}$, the bundle

$$
\pi_{p}^{p+1}: J^{p+1}(X, Y) \longrightarrow J^{p}(X, Y)
$$

has a natural affine structure, and we denote by $\mathcal{F}_{p}^{p+1}(a)$ the fiber $\left(\pi_{p}^{p+1}\right)^{-1}(a)$, for $a \in J^{p}(X, Y)$. Given a submanifold $A \subseteq J^{p}(X, Y)$ of class $C^{r}, r \geq 1$, we define

$\tilde{A} \stackrel{\text { def }}{=}\left\{j_{x}^{p+1} f \in J^{p+1}(X, Y): j^{p} f\right.$ is not transverse to $A$ at $\left.x\right\} \subseteq J^{p+1}(X, Y)$.

If $j_{x}^{p} f$ is an element of $J^{p}(X, Y)$ and $0 \leq k \leq p-1$, we define the vector subspace $E^{k}\left(j_{x}^{p} f\right)$ by

$$
E^{k}\left(j_{x}^{p} f\right) \stackrel{\text { def }}{=} d\left(j_{x}^{k} f\right)\left(T_{x} X\right) \subseteq T_{j_{x}^{k}}\left(J^{k}(X, Y)\right)
$$


Here $d\left(j_{x}^{k} f\right)$ is the tangent map at $x$ of $j^{k} f: X \rightarrow J^{k}(X, Y)$. Note that the subspace $E^{k}\left(j_{x}^{p} f\right)$ depends just on $j_{x}^{k+1} f$ and that its dimension is always equal to $\operatorname{dim} X$. We also extend the definition to $k=-1$ in a trivial way by setting $E^{-1}\left(j_{x}^{p} f\right)=T_{x} X$. We have

$$
\tilde{A}=\left\{z \in J^{p+1}(X, Y): T_{\pi_{p}^{p+1} z} A+E^{p}(z) \subsetneq T_{\pi_{p}^{p+1} z} J^{p}(X, Y)\right\} .
$$

Conjecture 3.1. The set $\tilde{A}$ is a countable union of submanifolds of codimension more than $n=\operatorname{dim} X$.

This conjecture is stated as a lemma in [8], but not proved. The statement is obvious when the codimension $c$ of $A$ is larger than $n$, so we assume from now on that $c \leqslant n$. We will use the notation

$$
\tilde{A}_{a}=\tilde{A} \cap \mathcal{F}_{p}^{p+1}(a) .
$$

We say that the point $a \in A$ is degenerate if

$$
\pi_{p-1}^{p}\left(T_{a} A\right)+E^{p-1}(a) \subsetneq T_{\pi_{p-1}^{p} a} J^{p-1}(X, Y) .
$$

(for $p=0$ the map $\pi_{-1}^{0}$ is the projection from $J^{0}(X, Y)$ to $X$ ). If $a$ is degenerate, then $\tilde{A}_{a}=\mathcal{F}_{p}^{p+1}(a)$. The manifold $A$ can be decomposed as the disjoint union $A=A_{0} \cup A_{1}$, where $A_{0}$ is the set of degenerate points of $A$ and $A_{1}$ is the set of non-degenerate points (by definition, the other points). The set $A_{1}$ is an open submanifold of $A$, hence it is itself a submanifold of $J^{p}(X, Y)$, and

$$
\tilde{A}=\left(\pi_{p}^{p+1}\right)^{-1}\left(A_{0}\right) \cup \tilde{A_{1}} \text {. }
$$

Let us first treat the special case where $A=A_{1}$ (we then say that $A$ is nondegenerate). Note that this condition holds for example if $A$ is transverse to the fibers of the projection $\pi_{p-1}^{p}$. This condition also holds when $p=0$. The following result also implies Lemma 1.2:

Theorem 3.2. Let $A$ be a non-degenerate $C^{r}$ submanifold in $J^{p}(X, Y)$. Then $\tilde{A}$ is a countable union of $C^{r-1}$ submanifolds of codimension larger than $n=\operatorname{dim} X$ in $J^{p+1}(X, Y)$.

This result implies that $\tilde{A}_{1}$ is a countable union of submanifolds of codimension at least $n+1$. In order to prove the conjecture, we would also need to prove that the manifold $A_{0}$ has codimension $n+1$. We are not able to prove this statement in the general case, hence we will restrict to the analytic case. We say that the submanifold $A \subseteq J^{p}(X, Y)$ is analytic if for every $a=j_{x}^{p} f \in A$ there exist charts $\psi_{X}$ and $\psi_{Y}$ on $X$ and $Y$, respectively defined on a neighborhood of $x$ in $X$ and a 
neighborhood of $f(x)$ in $Y$, such that the induced chart $\psi$ on $J^{p}(X, Y)$, defined on a neighborhood $U_{a}$ of $a$, makes $A$ analytic, i.e.

$$
\psi\left(A \cap U_{a}\right)=\bigcap_{i} F_{i}^{-1}(0)
$$

for a finite family of analytic functions $F_{i}: \psi\left(U_{a}\right) \rightarrow \mathbb{R}$. When $A$ is analytic, we manage to study $A_{0}$ by recurrence using Theorem 3.2, and obtain:

Theorem 3.3. Let $A$ be an analytic submanifold of $J^{p}(X, Y)$. Then $\tilde{A}$ is a countable union of $C^{r-1}$-submanifolds of codimension larger than $n=\operatorname{dim} X$ in $J^{p+1}(X, Y)$.

\subsection{The non-degenerate case}

We assume here that the $C^{r}$-manifold $A \subset J^{p}(X, Y)$ is non-degenerate, which means that

$$
\pi_{p-1}^{p}\left(T_{a} A\right)+E^{p-1}(a)=T_{\pi_{p-1}^{p} a} J^{p-1}(X, Y)
$$

for each $a \in A$, and we prove Theorem 3.2. To study the set $\tilde{A}_{a}$ we define, more generally, the set

$$
Z_{a, V}=\left\{\hat{a} \in \mathcal{F}_{p}^{p+1}(a): V+E^{p}(\hat{a}) \subsetneq T_{a} J^{p}(X, Y)\right\} \subseteq \mathcal{F}_{p}^{p+1}(a)
$$

associated to a point $a \in J^{p}(X, Y)$ and a subspace $V \subset T_{a} J^{p}(X, Y)$. Then, we have

$$
\tilde{A}_{a}=Z_{a, T_{a} A} .
$$

We decompose $Z_{a, V}$ as

$$
Z_{a, V}=\bigcup_{r=\operatorname{dim} V}^{\operatorname{dim} J^{p}(X, Y)-1} Z_{a, V}^{r}
$$

where

$$
Z_{a, V}^{r} \stackrel{\text { def }}{=}\left\{\hat{a} \in\left(\mathcal{F}_{p}^{p+1}\right)(a): \operatorname{dim}\left(V+E^{p}(\hat{a})\right)=r\right\} .
$$

This decomposition obviously yields a decomposition $\tilde{A}=\cup \tilde{A}^{r}$, where

$$
\tilde{A}_{a}^{r}:=\tilde{A}^{r} \cap \mathcal{F}_{p}^{p+1}(a)=Z_{a, T_{a} A}^{r} .
$$

The following result implies that $\tilde{A}^{r}$ is a $C^{r-1}$-submanifold of codimension at least $n+1-c$ in $\left(\pi_{p}^{p+1}\right)^{-1}(A)$, hence a submanifold of codimension at least $n+1$ in $J^{p+1}(X, Y)$, which proves Theorem 3.2. 
Proposition 3.4. Let $a \in J^{p}(X, Y)$ and $V$ be a vector subspace of $T_{a} J^{p}(X, Y)$ of dimension $m$ and codimension $c \geq 1$ such that

$$
\pi_{p-1}^{p}(V)+E^{p-1}(a)=T_{\pi_{p-1}^{p} a} J^{p-1}(X, Y) .
$$

We have:

$$
\operatorname{codim}_{\mathcal{F}_{p}^{p+1}(a)} Z_{a, V} \geq n+1-c .
$$

More precisely, the set $Z_{a, V}^{r}$ is locally contained in the preimage $F_{a, V}^{-1}(0)$ of an algebraic submersion

$$
F_{a, V}: \mathcal{F}_{p}^{p+1}(a) \longrightarrow \mathbb{R}^{\theta}
$$

whose coefficients depend smoothly on $(a, V)$, with $\theta=n+1-c+2\left(\operatorname{dim} J^{p}(X, Y)-\right.$ $1-r)$.

Proof. Since the result is of local nature, we can suppose without loss of generality that the jet bundles are trivialized. Hence,

$$
J^{p+1}(X, Y)=J^{p}(X, Y) \times \mathcal{F}_{p}^{p+1}
$$

where $\mathcal{F}_{p}^{p+1}$ is the fiber of the projection $\pi_{p}^{p+1}: J^{p+1}(X, Y) \rightarrow J^{p}(X, Y)$. We have thus the identification $\mathcal{F}_{p}^{p+1}(a) \cong \mathcal{F}_{p}^{p+1}$. Hence the sets $Z_{a, V}^{r}$ can be regarded as subsets of $\mathcal{F}_{p}^{p+1}$ : denoting by $z$ the elements of $\mathcal{F}_{p}^{p+1}$, we have

$$
Z_{a, V}^{r}=\left\{z \in \mathcal{F}_{p}^{p+1}: \operatorname{dim}\left(V+E^{p}(a, z)\right)=r\right\} .
$$

We assume from now on that $m \leq r \leq \operatorname{dim} J^{p}(X, Y)-1$. Let us pick, for any $z$ in $\mathcal{F}_{p}^{p+1}$, a function $f_{z}$ such that

$$
j_{x}^{p+1} f_{z}=(a, z) .
$$

Here $x \in X$ is the basis-point of $a$. Let us also choose a basis $v_{1}, \ldots, v_{m}$ of $V$. Let us call $M_{a, V}(z)$ (or just $\left.M(z)\right)$ the matrix whose columns are, in the order, the following vectors (belonging to $T_{a} J^{p}(X, Y)$ )

$$
v_{1}, \ldots, v_{m}, \partial_{x_{1}} j_{x}^{p} f_{z}, \ldots, \partial_{x_{n}} j_{x}^{p} f_{z}
$$

expressed in a convenient basis of $T_{a} J^{p}(X, Y)$ which we shall make explicit shortly. Note that the vectors $\partial_{x_{j}} j_{x}^{p} f_{z}, 1 \leq j \leq n$ form a basis of $E^{p}(a, z)$. It is then clear that

$$
z \in Z_{a, V}^{r} \Leftrightarrow \operatorname{rank} M_{a, V}(z)=r,
$$

or equivalently

$$
z \in Z_{a, V}^{r} \Leftrightarrow \begin{cases}\operatorname{det} N(z)=0 & \forall \text { square submatrix } N \text { of } M_{a, V} \text { of size } r+1 \\ \operatorname{det} N(z) \neq 0 & \text { for some square submatrix } N \text { of size } r .\end{cases}
$$


We will now study more precisely these equations with the help of an appropriate system of local coordinates. Locally, we have the identifications

$$
\begin{aligned}
J^{p}(X, Y) & =J^{p-1}(X, Y) \times \mathcal{F}_{p-1}^{p} \\
J^{p+1}(X, Y) & =J^{p-1}(X, Y) \times \mathcal{F}_{p-1}^{p} \times \mathcal{F}_{p}^{p+1},
\end{aligned}
$$

and both $\mathcal{F}_{p}^{p+1}$ and $\mathcal{F}_{p-1}^{p}$ can be identified to real vector spaces. More precisely, we fix once and for all local coordinates $x_{1}, \ldots, x_{n}$ and $y^{1}, \ldots, y^{q}$ on $X$ and $Y$ respectively; this induces the identification $\mathcal{F}_{p}^{p+1} \cong \mathbb{R}^{\operatorname{dim} \mathcal{F}_{p}^{p+1}}=\mathbb{R}^{q\left(\begin{array}{c}n+p \\ n-1\end{array}\right)}$ via the isomorphism

$$
\begin{aligned}
\left(y_{\alpha}^{s}\right)_{1 \leq s \leq q,|\alpha|=p+1}: \mathcal{F}_{p}^{p+1} & \rightarrow \mathbb{R}^{q\left(\begin{array}{c}
n+p \\
n-1
\end{array}\right)} \\
j_{x}^{p} f & \mapsto\left(\partial_{\alpha} f^{s}(x)\right)_{1 \leq s \leq q,|\alpha|=p+1} .
\end{aligned}
$$

Here $f^{s}=y^{s} \circ f$ is the $s$-th component of $f, \alpha=\left(\alpha_{1}, \ldots, \alpha_{n}\right)$ is a multi-index in $\mathbb{N}^{n}$ of length $|\alpha|=p+1$ and $\partial_{\alpha}=\partial_{x_{1}}^{\alpha_{1}} \ldots \partial_{x_{n}}^{\alpha_{n}}$ stands for the associated partial derivative. Note that for the isomorphism to be rigorously defined, one should specify an order on the set of the involved pairs $(s, \alpha)$. Since this order will not play any role in the sequel, we do not specify it.

Concerning $\mathcal{F}_{p-1}^{p}$, we have the analogous identification $\mathcal{F}_{p-1}^{p} \cong \mathbb{R}^{\operatorname{dim} \mathcal{F}_{p-1}^{p}=}$ $\mathbb{R}^{q\left(\begin{array}{c}n+p-1 \\ n-1\end{array}\right)}$ via the isomorphism

$$
\begin{aligned}
\left(y_{\alpha}^{s}\right)_{1 \leq s \leq q,|\alpha|=p}: \mathcal{F}_{p-1}^{p} & \rightarrow \mathbb{R}^{q\left(\begin{array}{c}
n+p-1 \\
n-1
\end{array}\right)} \\
j_{x}^{p} f & \mapsto\left(\partial_{\alpha} f^{s}(x)\right)_{1 \leq s \leq q,|\alpha|=p} .
\end{aligned}
$$

Here the order on the pairs $(s, \alpha)$ will play an important role. For reasons which will become clear in Lemma 3.8, we adopt the following lexicographic order: ${ }^{2}$ if $s, s^{\prime} \in\{1, \ldots, q\}$ and $\alpha=\left(\alpha_{1}, \ldots, \alpha_{n}\right), \alpha^{\prime}=\left(\alpha_{1}^{\prime}, \ldots, \alpha_{n}^{\prime}\right)$ are multi-indices of length $p$, the variable $y_{\alpha}^{s}$ strictly precedes the variable $y_{\alpha^{\prime}}^{s^{\prime}}$ if and only if

$$
s>s^{\prime} \text { or }\left(s=s^{\prime} \text { and } \exists k \geq 1:\left\{\begin{array}{l}
\alpha_{h}=\alpha_{h}^{\prime} \\
\alpha_{k}>\alpha_{k}^{\prime}
\end{array} \quad \text { for all } 1 \leq h \leq k-1\right)\right. \text {. }
$$

2 What really matters for our purposes is the lexicographic order with respect to $\alpha$ at fixed $s$. There are several orders satisfying this condition, but for the sake of definiteness we adopt the one described in (3.9). 
Summing up the above paragraphs, we will regard $\left(y_{\alpha}^{s}\right)_{1 \leq s \leq q,|\alpha|=p+1}$ and $\left(y_{\alpha}^{s}\right)_{1 \leq s \leq q,|\alpha|=p}$ as coordinates respectively on $\mathcal{F}_{p}^{p+1}$ and $\mathcal{F}_{p-1}^{p}$, compatible with the affine structure of these spaces. The coordinates on $\mathcal{F}_{p-1}^{p}$ are ordered according to (3.9). Since we denoted by $z$ the elements of $\mathcal{F}_{p}^{p+1}$, we have

$$
z=\left(y_{\alpha}^{s}\right)_{1 \leq s \leq q,|\alpha|=p+1} \in \mathcal{F}_{p}^{p+1} \cong \mathbb{R}^{\operatorname{dim} \mathcal{F}_{p}^{p+1}} .
$$

Let us now write more explicitly the vectors $\partial_{x_{j}} j_{x}^{p} f_{z}$ appearing in (3.7). According to the decomposition (3.8), an arbitrary $p$-jet $j_{x}^{p} f$ writes as

$$
j_{x}^{p} f=\left(j_{x}^{p-1} f,\left(\partial_{\alpha} f^{s}(x)\right)_{1 \leq s \leq q,|\alpha|=p}\right) \quad \in J^{p-1}(X, Y) \times \mathcal{F}_{p-1}^{p} .
$$

The vectors $\partial_{x_{j}} j_{x}^{p} f_{z}$ are elements of the vector space $T_{a} J^{p}(X, Y)=T_{\pi_{p-1}^{p} a} J^{p-1}(X, Y) \times$ $\mathcal{F}_{p-1}^{p}$. Taking the derivative of (3.10) with respect to $x_{j}$ we get:

$$
\begin{aligned}
\partial_{x_{j}} j_{x}^{p} f_{z} & =\left(\partial_{x_{j}} j_{x}^{p-1} f_{z},\left(\partial_{\alpha+\delta_{j}} f^{s}(x)\right)_{1 \leq s \leq q,|\alpha|=p}\right) \\
& =\left(\partial_{x_{j}} j_{x}^{p-1} f_{z},\left(y_{\alpha+\delta_{j}}^{s}\right)_{1 \leq s \leq q,|\alpha|=p}\right)
\end{aligned}
$$

where $\delta_{j}$ is the multi-index $(0, \ldots, 0, \underbrace{1}_{j \text {-th }}, 0, \ldots, 0) \in \mathbb{N}^{n}$. Note that the component $\partial_{x_{j}} j_{x}^{p-1} f_{z}$ depends just on partial derivatives of order one of $j^{p-1} f_{z}$ at $x$, i.e. it depends just on $j_{x}^{p} f_{z}=a$. This justifies the following notation:

$$
e_{j}(a) \stackrel{\text { def }}{=} \partial_{x_{j}} j_{x}^{p-1} f_{z}, \quad 1 \leq j \leq n
$$

Note that

$$
\operatorname{Span}\left\{e_{1}(a), \ldots, e_{n}(a)\right\}=E^{p-1}(a) .
$$

We have:

$$
\partial_{x_{j}} j_{x}^{p} f_{z}=\left(e_{j}(a),\left(y_{\alpha+\delta_{j}}^{s}\right)_{1 \leq s \leq q,|\alpha|=p}\right) .
$$

We can now write the matrix $M_{a, V}(z)$ in the basis of

$$
T_{a} J^{p}(X, Y)=T_{\pi_{p-1}^{p} a} J^{p-1}(X, Y) \times \mathcal{F}_{p-1}^{p}
$$


obtained as the juxtaposition of an arbitrary basis of $T_{\pi_{p-1}^{p} a} J^{p-1}(X, Y)$ and of the basis $\left(y_{\alpha}^{s}\right)_{1 \leq s \leq q,|\alpha|=p}$ of $\mathcal{F}_{p-1}^{p}$ in the order which has been specified before:

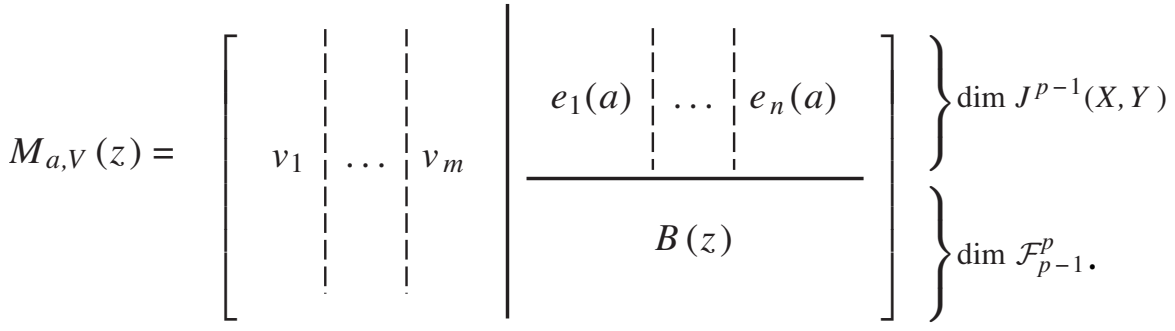

Here the vectors $v_{1}, \ldots, v_{m}$ are a basis of $V$ and the vectors $e_{1}(a), \ldots, e_{n}(a)$, which have been defined before, form a basis of $E^{p-1}(a)$. Finally, the block $B(z)$ depends just on $z$ and is given by

$$
B(z)=\left[\begin{array}{ccccc}
\vdots & \vdots & \vdots & \vdots & \vdots \\
y_{\alpha+\delta_{1}}^{s} & y_{\alpha+\delta_{2}}^{s} & \cdots & y_{\alpha+\delta_{n-1}}^{s} & y_{\alpha+\delta_{n}}^{s} \\
\vdots & \vdots & \vdots & \vdots & \vdots
\end{array}\right] \leftarrow \text { row corresponding to } y_{\alpha}^{s}
$$

where the rows are ordered according to (3.9).

For later use, note that the first $m$ columns of $M(z)$ are linearly independent, as well as the first $\operatorname{dim} J^{p-1}(X, Y)$ rows, as it follows from the hypothesis (3.6). Indeed, the first $m$ columns are clearly independent because they represent a basis of $V$. The fact that the first $\operatorname{dim} J^{p-1}(X, Y)$ rows are independent is equivalent to the assumption $\pi_{p-1}^{p}(V)+E^{p-1}(a)=T_{\pi_{p-1}^{p} a} J^{p-1}(X, Y)$.

An intermede of linear algebra. We prove, for an arbitrary matrix, the existence of a non-singular square submatrix of maximum rank satisfying some special conditions.

Let $M$ be an arbitrary $m \times n$-matrix with real entries. Only in this intermede, $m$ and $n$ are arbitrary integers $\geq 1$, with no relation with the values assumed by the same symbols in the rest of the paper.

Let us establish some notation for submatrices of $M$. The rows of $M$ are $\mathcal{R}(M)=\{1,2, \ldots, m\}$ and its columns are $\mathcal{C}(M)=\{1,2, \ldots, n\}$. A submatrix $N$ of $M$ is determined by its rows

$$
\mathcal{R}(N) \subseteq\{1,2, \ldots, m\}
$$

and its columns

$$
\mathcal{C}(N) \subseteq\{1,2, \ldots, n\} .
$$

We denote $|\mathcal{R}(N)|$ and $|\mathcal{C}(N)|$ their cardinality. We also denote by $i_{1}(N), i_{2}(N), \ldots$ $\ldots, i_{|\mathcal{R}(N)|}(N)$ the elements of $\mathcal{R}(N)$, and we always assume that the indices are 
chosen in such a way that

$$
i_{1}(N)<i_{2}(N)<\ldots<i_{|\mathcal{R}(N)|}(N) .
$$

Similarly, we denote $\mathcal{C}(N)=\left\{j_{1}(N), \ldots, j_{|\mathcal{C}(N)|}(N)\right\}$ with

$$
j_{1}(N)<j_{2}(N)<\ldots<j_{|\mathcal{C}(N)|}(N) .
$$

Given two sub matrices $N_{1}$ and $N_{2}$, we say that $N_{1} \preceq_{\mathcal{R}} N_{2}$ if the rows of $N_{1}$ come "before" the rows of $N_{2}$; more rigorously,

$$
N_{1} \preceq_{\mathcal{R}} N_{2} \stackrel{\text { def }}{\Longleftrightarrow}\left|\mathcal{R}\left(N_{1}\right)\right| \leq\left|\mathcal{R}\left(N_{2}\right)\right| \text { and } i_{k}\left(N_{1}\right) \leq i_{k}\left(N_{2}\right) \quad \forall 1 \leq k \leq\left|\mathcal{R}\left(N_{1}\right)\right| .
$$

We also give the analogous definition for columns:

$$
N_{1} \preceq_{\mathcal{C}} N_{2} \stackrel{\text { def }}{\Longleftrightarrow}\left|\mathcal{C}\left(N_{1}\right)\right| \leq\left|\mathcal{C}\left(N_{2}\right)\right| \text { and } i_{k}\left(N_{1}\right) \leq i_{k}\left(N_{2}\right) \quad \forall 1 \leq k \leq\left|\mathcal{C}\left(N_{1}\right)\right| \text {. }
$$

Note that $\preceq_{\mathcal{R}}$ and $\preceq_{\mathcal{C}}$ are preorders (i.e. reflexive and transitive) but not partial orders in general. We write $N_{1} \prec_{\mathcal{R}} N_{2}$ if $N_{1} \preceq_{\mathcal{R}} N_{2}$ and $N_{1} \neq N_{2}$. We define $N_{1} \prec_{\mathcal{C}} N_{2}$ similarly.

It turns out that, when restricted to the set of square submatrices of rank equal to rank $M$, the relations $\preceq_{\mathcal{R}}$ and $\preceq_{\mathcal{C}}$ admit a unique common minimal element, in a sense made precise by the following lemma.

Lemma 3.5. Let $M$ be an $m \times n$-matrix. There exists a submatrix $M^{*}$ of $M$ such that rank $M^{*}=\operatorname{rank} M$ and which is minimal in the following sense: any submatrix $N$ with $\operatorname{rank} N=\operatorname{rank} M$ satisfies

$$
M^{*} \preceq_{\mathcal{R}} N \text { and } M^{*} \preceq_{\mathcal{C}} N .
$$

The submatrix $M^{*}$ is uniquely defined by this condition, and it is a square matrix.

It is easy to check that if such a submatrix $M^{*}$ exists, then it is unique and square. Thus we just focus on the existence. We will prove existence in a somehow constructive way, by giving a procedure for finding $M^{*}$. In fact, we will give two different procedures and we will show that they yield the same submatrix; as a consequence, this submatrix will satisfy the conditions demanded to $M^{*}$.

As a first intermediate step, let us describe two constructions which allow to associate to $M$ two special (non-square in general) submatrices. We call these two sub matrices $V(M)$ and $H(M)$. Here $V$ stands for vertical and $H$ for horizontal.

Let us first describe how to construct $V(M)$ : it is uniquely defined by the properties

$$
\begin{aligned}
\mathcal{C}(V(M))= & \mathcal{C}(M)=\{1, \ldots, n\} \\
i \in \mathcal{R}(V(M)) \Leftrightarrow & \text { the } i \text {-th row of } M \text { is linearly independent from } \\
& \text { the first } i-1 \text { rows of } M .
\end{aligned}
$$


(If $i=0$, we mean that $i \in \mathcal{R}(V(M))$ if and only if the first row is not identically zero.) This procedure ensures that $V(M)$ is minimal with respect to rows among submatrices of $M$ of maximal rank. More precisely, $V(M)$ satisfies

$\operatorname{rank} V(M)=\operatorname{rank} M$ and $V(M) \preceq_{\mathcal{R}} N \forall N$ submatrix of $M$ with $\operatorname{rank} N=\operatorname{rank} M$.

The construction of $H(M)$ is the same as for $V(M)$, but with the roles of rows and columns inverted. More precisely,

$$
\begin{aligned}
\mathcal{R}(H(M))= & \mathcal{R}(M)=\{1, \ldots, m\} \\
j \in \mathcal{C}(H(M)) \Leftrightarrow & \text { the } j \text {-th row of } M \text { is linearly independent from } \\
& \text { the first } j-1 \text { rows of } M .
\end{aligned}
$$

Analogously,

$\operatorname{rank} H(M)=\operatorname{rank} M$ and $H(M) \preceq_{\mathcal{C}} N \forall N$ submatrix of $M$ with $\operatorname{rank} N=\operatorname{rank} M$.

Now that we have introduced the two constructions, we can iterate them. In particular, we can consider $H V(M)$ and $V H(M)$. We regard them as submatrices of $M$. By construction, they are square non-singular submatrices of size equal to rank $M$. In fact, it turns out that they coincide, and they are the submatrix $M^{*}$ which we are looking for. More precisely, the following two claims are true:

(i) $H V(M)=V H(M)$;

(ii) the matrix $M^{*}:=H V(M)=V H(M)$ satisfies the conditions required in the statement.

Proof of (i). We want to prove that $\mathcal{R}(H V(M))=\mathcal{R}(V H(M))$ and $\mathcal{C}(H V(M))=$ $\mathcal{C}(V H(M))$. As already pointed out, $H V(M)$ and $V H(M)$ are square submatrices of equal size (equal to rank $M)$. Hence it suffices to prove that $\mathcal{R}(V H(M)) \subseteq$ $\mathcal{R}(H V(M))$ and that $\mathcal{C}(H V(M)) \subseteq \mathcal{C}(V H(M))$. We focus just on the first inclusion, the second being analogous.

By the properties of the constructions $H$ and $V$ described above, we have:

$$
\begin{aligned}
k \in \mathcal{R}(V H(M)) \Rightarrow & \text { the } k \text {-th row of } H(M) \text { is linearly independent } \\
& \text { from the first } k-1 \text { rows of } H(M) \\
\Rightarrow & \text { the } k \text {-th row of } M \text { is linearly independent } \\
& \text { from the first } k-1 \text { rows of } M \\
\Rightarrow & k \in \mathcal{R}(V(M))=\mathcal{R}(H V(M))
\end{aligned}
$$

as desired.

Proof of (ii). Let $N$ be a sub matrix of $M$ with $\operatorname{rank} N=\operatorname{rank} M$. By the properties of $V(M), V(M) \preceq \mathcal{R} N$. From $M^{*}=H V(M)$ we deduce $\mathcal{R}\left(M^{*}\right)=$ $\mathcal{R}(H V(M))=\mathcal{R}(V(M))$ and thus $M^{*} \preceq_{\mathcal{R}} N$ as well. The proof of $M^{*} \preceq_{\mathcal{C}} N$ is similar. 
Let us emphasize the following characterization of $M^{*}$ which follows directly from the proof above:

$i \in \mathcal{R}\left(M^{*}\right) \Leftrightarrow$ the $i$-th row of $M$ does not belong to the linear span of the first $i-1$ rows of $M$.

$j \in \mathcal{C}\left(M^{*}\right) \Leftrightarrow$ the $j$-th column of $M$ does not belong to the linear span of the first $j-1$ columns of $M$.

End of the proof of Proposition 3.4. We fix $r$ between $m$ and $\operatorname{dim} J^{p}(X, Y)-1$ and assume that $Z_{a, V}^{r}$ is not empty (otherwise we have nothing to prove). Let $z_{0}$ be an arbitrary element of $Z_{a, V}^{r}$. Our goal will now be to find $\theta$ different square submatrices $\left[M_{a, V}(z)\right]_{i}$ of size $r+1$ in $M_{a, V}(z)$ such that the equations $\operatorname{det}\left[M_{a, V}(z)\right]_{i}=0$ are independent near $z_{0}$. The functions $z \longmapsto \operatorname{det}\left[M_{a, V}(z)\right]_{i}$ are then the components of the map $F_{a, V}(z)$ mentioned in Proposition 3.4. These functions are clearly polynomials in $z$, with coefficients depending smoothly on $a$ and $V$. We omit from now on to explicitly mention $a$ and $V$, and note $M(z)$ instead of $M_{a, V}(z)$.

Let $M_{z_{0}}^{*}$ be the square submatrix of size $r$ associated to $M\left(z_{0}\right)$ by the Lemma 3.5. We take the notational convention that the symbol $M_{z_{0}}^{*}$ without further specifications stands for a pattern of rows and columns, i.e. $M_{z_{0}}^{*}$ is the datum $\left(\mathcal{R}\left(M_{z_{0}}^{*}\right)\right.$, $\left.\mathcal{C}\left(M_{z_{0}}^{*}\right)\right)$. We can also identify $M_{z_{0}}^{*}$ to a matrix-valued function of $z$, but in this case we explicitly write $M_{z_{0}}^{*}(z)$ or $M_{z_{0}}^{*}(\cdot)$. This is in order to avoid ambiguities and distinguish, for instance, between $M_{z_{0}}^{*}$ and $M_{z_{0}}^{*}\left(z_{0}\right)$. We adopt the same convention for all the submatrices encountered below, such as $\hat{M}_{z_{0}}, M_{z_{0}, \#(i, j)}^{*}$, etc., which we shall define shortly.

We have

$$
\operatorname{det} M_{z_{0}}^{*}\left(z_{0}\right) \neq 0 \quad \text { and } \quad \operatorname{rank} M_{z_{0}}^{*}\left(z_{0}\right)=\operatorname{rank} M\left(z_{0}\right)=r .
$$

Let us call $\hat{M}_{z_{0}}$ the submatrix whose rows and columns are exactly the ones not appearing in $M_{z_{0}}^{*}$, i.e.

$\mathcal{R}\left(\hat{M}_{z_{0}}\right)=\left\{1, \ldots, \operatorname{dim} J^{p}(X, Y)\right\} \backslash \mathcal{R}\left(M_{z_{0}}^{*}\right), \quad \mathcal{C}\left(\hat{M}_{z_{0}}\right)=\{1, \ldots, m+n\} \backslash \mathcal{C}\left(M_{z_{0}}^{*}\right)$.

As already mentioned above, the first $m$ columns of $M$ are linearly independent as well as the first $\operatorname{dim} J^{p-1}(X, Y)$-rows. By the characterization (3.14) we deduce that $\hat{M}_{z_{0}}$ is entirely contained in the bottom-right block of $M$, i.e. it is a submatrix of $B$ :

$$
\begin{aligned}
\mathcal{R}\left(\hat{M}_{z_{0}}\right) \subseteq \mathcal{R}(B) & =\left\{\operatorname{dim} J^{p-1}(X, Y)+1, \ldots, \operatorname{dim} J^{p}(X, Y)\right\} \\
\mathcal{C}\left(\hat{M}_{z_{0}}\right) \subseteq \mathcal{C}(B) & =\{m+1, \ldots, m+n\} .
\end{aligned}
$$

Let us denote $M_{z_{0}, \#(i, j)}^{*}$ the submatrix obtained by adding to $M_{z_{0}}^{*}$ the $i$-th row and the $j$-th column of $M$, i.e.

$$
\mathcal{R}\left(M_{z_{0}, \#(i, j)}^{*}\right)=\mathcal{R}\left(M_{z_{0}}^{*}\right) \cup\{i\}, \quad \mathcal{C}\left(M_{z_{0}, \#(i, j)}^{*}\right)=\mathcal{C}\left(M_{z_{0}}^{*}\right) \cup\{j\} .
$$


We are interested in the case when $(i, j)$ belongs to $\mathcal{R}\left(\hat{M}_{z_{0}}\right) \times \mathcal{C}\left(\hat{M}_{z_{0}}\right)$. In this case the submatrix $M_{z_{0}, \#(i, j)}^{*}$ is a square submatrix of size $r+1$. We are in the following situation:

$$
\left\{\begin{array}{l}
\operatorname{det} M_{z_{0}}^{*}\left(z_{0}\right) \neq 0 \\
\operatorname{det} M_{z_{0}, \#(i, j)}^{*}\left(z_{0}\right)=0 \quad \forall(i, j) \in \mathcal{R}\left(\hat{M}_{z_{0}}\right) \times \mathcal{C}\left(\hat{M}_{z_{0}}\right) .
\end{array}\right.
$$

Proposition 3.4 then follows from the following two lemmas:

Lemma 3.6. If $\left(i_{1}, j_{1}\right), \ldots,\left(i_{\theta}, j_{\theta}\right)$ are pairwise distinct pairs in $\mathcal{R}\left(\hat{M}_{z_{0}}\right) \times \mathcal{C}\left(\hat{M}_{z_{0}}\right)$ such that

$$
i_{1} \leq i_{2} \leq \cdots \leq i_{\theta} \quad \text { and } \quad j_{1} \leq j_{2} \leq \cdots \leq j_{\theta},
$$

then the differentials evaluated at $z_{0}$

$$
d_{z_{0}} \operatorname{det} M_{z_{0}, \#\left(i_{1}, j_{1}\right)}^{*}(\cdot), \ldots, d_{z_{0}} \operatorname{det} M_{z_{0}, \#\left(i_{\theta}, j_{\theta}\right)}^{*}(\cdot)
$$

are linearly independent.

Lemma 3.7. For $\theta=n+1-c+2\left(\operatorname{dim} J^{p}(X, Y)-1-r\right)$, there exist pairwise distinct pairs as above.

Proof of Lemma 3.7. One may for instance consider the pairs of indices successively encountered along the following "path" in the matrix $\hat{M}_{z_{0}}$ : starting from the upper-left corner of the matrix, and then moving horizontally along the first row until the upper-right corner, and then moving vertically along the last column until the bottom-right corner. It is clear that the pairs of indices successively encountered along this path satisfy the condition (3.15). Their number is the "semi-perimeter" of the matrix, or more rigorously $\left|\mathcal{R}\left(\hat{M}_{z_{0}}\right)\right|+\left|\mathcal{C}\left(\hat{M}_{z_{0}}\right)\right|-1$. Recalling the definition of $\hat{M}_{z_{0}}$, this is the same as

$$
\begin{aligned}
|\mathcal{R}(M)|-\left|\mathcal{R}\left(M_{z_{0}}^{*}\right)\right|+|\mathcal{C}(M)|-\left|\mathcal{C}\left(M_{z_{0}}^{*}\right)\right|-1 & =\left(\operatorname{dim} J^{p}(X, Y)-r\right)+(m+n-r)-1 \\
& =n+1-c+2\left(\operatorname{dim} J^{p}(X, Y)-1-r\right)
\end{aligned}
$$

This ends the proof of Lemma 3.7.

Proof of Lemma 3.6. For $(i, j) \in \mathcal{R}(B) \times \mathcal{C}(B)$, we recall that $i$ is the index of a line of $B$, hence it corresponds to a coordinate $y_{\alpha(i)}^{s(i)}$ of $\mathcal{F}_{p-1}^{p}$, while $j$ is an integer between $m+1$ and $m+n$. Then, the coefficient of the matrix $B(z)$ at line $i$ and column $j$ is just $y_{\alpha(i)+\delta_{j-m}}^{s(i)}$. It is a component, that we denote by $z_{[i, j]}$, of $z$. Note however that the same component of $z$ may appear at several different places in the matrix $B(z)$. It can happen that $z_{[i, j]}=z_{\left[i^{\prime}, j^{\prime}\right]}$ with $(i, j) \neq\left(i^{\prime}, j^{\prime}\right)$, it is the case when $s(i)=s\left(i^{\prime}\right)$ and $\alpha(i)+\delta_{j-m}=\alpha\left(i^{\prime}\right)+\delta_{j^{\prime}-m}$. Our order on the coordinates allows us to overcome this difficulty thanks to the following lemma: 
Lemma 3.8. Let $(i, j)$ and $(h, k)$ belong to $\mathcal{R}(B) \times \mathcal{C}(B)$, and satisfy

$$
h \geq i, k \geq j,(i, j) \neq(h, k) .
$$

Then, $z_{[h, k]} \neq z_{[i, j]}$ and, if $(i, j)$ and $(h, k)$ belong to $\mathcal{R}\left(\hat{M}_{z_{0}}\right) \times \mathcal{C}\left(\hat{M}_{z_{0}}\right)$, then

$$
\begin{aligned}
& \frac{\partial}{\partial z_{[i, j]}} \operatorname{det} M_{z_{0}, \#(i, j)}^{*}\left(z_{0}\right)= \pm \operatorname{det} M_{z_{0}}^{*}\left(z_{0}\right) \neq 0 \\
& \frac{\partial}{\partial z_{[h, k]}} \operatorname{det} M_{z_{0}, \#(i, j)}^{*}\left(z_{0}\right)=0 .
\end{aligned}
$$

Lemma 3.8 implies that $z_{\left[i_{1}, j_{1}\right]}, \ldots, z_{\left[i_{\theta}, j_{\theta}\right]}$ are pairwise distinct components of $z$, and that the square matrix

$$
\left[\begin{array}{ccc}
\frac{\partial}{\partial z_{\left[i_{1}, j_{1}\right]}} \operatorname{det} M_{z_{0}, \#\left(i_{1}, j_{1}\right)}^{*}\left(z_{0}\right) & \ldots & \frac{\partial}{\partial z_{\left[i_{\theta}, j_{\theta}\right]}} \operatorname{det} M_{z_{0}, \#\left(i_{1}, j_{1}\right)}^{*}\left(z_{0}\right) \\
\vdots & \ddots & \vdots \\
\frac{\partial}{\partial z_{\left[i_{1}, j_{1}\right]}} \operatorname{det} M_{z_{0}, \#\left(i_{\theta}, j_{\theta}\right)}^{*}\left(z_{0}\right) & \ldots & \frac{\partial}{\partial z_{\left[i_{\theta}, j_{\theta}\right]}} \operatorname{det} M_{z_{0}, \#\left(i_{\theta}, j_{\theta}\right)}^{*}\left(z_{0}\right)
\end{array}\right]
$$

has the form

$$
\left[\begin{array}{ccccc} 
\pm \operatorname{det} M_{z_{0}}^{*}\left(z_{0}\right) & 0 & 0 & \ldots & 0 \\
* & \pm \operatorname{det} M_{z_{0}}^{*}\left(z_{0}\right) & 0 & \ldots & 0 \\
* & * & \pm \operatorname{det} M_{z_{0}}^{*}\left(z_{0}\right) & \ldots & 0 \\
\vdots & \vdots & \vdots & \ddots & \vdots \\
* & * & * & \ldots \pm \operatorname{det} M_{z_{0}}^{*}\left(z_{0}\right)
\end{array}\right]
$$

hence it is invertible, since $\operatorname{det} M_{z_{0}}^{*}\left(z_{0}\right) \neq 0$. This proves Lemma 3.6, using Lemma 3.8 .

Proof of Lemma 3.8. Let us first prove that $z_{[i, j]} \neq z_{[h, k]}$. If $s(i) \neq s(h)$, then the conclusion holds. If $s(i)=s(h)$, then $\alpha(i) \geqslant \alpha(h)$ for the lexicographic order. On the other hand, the inequality $k \geqslant j$ implies that $\delta_{k-m} \leqslant \delta_{j-m}$ for the lexicographic order. These two inequalities do not sum to an equality because they are not both equalities (recall the hypothesis $(i, j) \neq(h, k))$, hence $\alpha(i)+\delta_{j-m} \neq \alpha(h)+\delta_{k-m}$, and then $z_{[i, j]} \neq z_{[h, k]}$.

To prove the equality

$$
\frac{\partial}{\partial z_{[h, k]}} \operatorname{det} M_{z_{0}, \#(i, j)}^{*}\left(z_{0}\right)=0,
$$


let us consider, for every $\left(h^{\prime}, k^{\prime}\right) \in\left(\mathcal{R}\left(M_{z_{0}, \#(i, j)}^{*}\right) \times \mathcal{C}\left(M_{z_{0}, \#(i, j)}^{*}\right)\right)$, the submatrix $N_{h^{\prime}, k^{\prime}}$ defined by

$$
\begin{aligned}
& \mathcal{R}\left(N_{h^{\prime}, k^{\prime}}\right)=\mathcal{R}\left(M_{z_{0}, \#(i, j)}^{*}\right) \backslash\left\{h^{\prime}\right\}=\left(\mathcal{R}\left(M_{z_{0}}^{*}\right) \cup\{i\}\right) \backslash\left\{h^{\prime}\right\} \\
& \mathcal{C}\left(N_{h^{\prime}, k^{\prime}}\right)=\mathcal{C}\left(M_{z_{0}, \#(i, j)}^{*}\right) \backslash\left\{k^{\prime}\right\}=\left(\mathcal{C}\left(M_{z_{0}}^{*}\right) \cup\{j\}\right) \backslash\left\{k^{\prime}\right\} .
\end{aligned}
$$

We have

$$
\frac{\partial}{\partial z_{[h, k]}} \operatorname{det} M_{z_{0}, \#(i, j)}^{*}\left(z_{0}\right)=\sum_{\left(h^{\prime}, k^{\prime}\right)} \pm \operatorname{det} N_{h^{\prime}, k^{\prime}}\left(z_{0}\right)
$$

where the actual sign is irrelevant and the sum is taken over all pairs $\left(h^{\prime}, k^{\prime}\right) \in$ $\left(\mathcal{R}\left(M_{z_{0}, \#(i, j)}^{*}\right) \times \mathcal{C}\left(M_{z_{0}, \#(i, j)}^{*}\right)\right)$ such that $z_{\left[h^{\prime}, k^{\prime}\right]}=z_{[h, k]}$.

We claim that each square matrix $N_{h^{\prime}, k^{\prime}}$ is singular, thus proving 3.17. In view of the definition of $M_{z_{0}}^{*}$ in Lemma 3.5, it is enough to observe that we can't have both $M_{z_{0}}^{*} \preceq_{\mathcal{R}} N_{h^{\prime}, k^{\prime}}$ and $M_{z_{0}}^{*} \preceq_{\mathcal{C}} N_{h^{\prime}, k^{\prime}}$. This would imply that we have both $h^{\prime} \leqslant i$ and $k^{\prime} \leqslant j$ and then that $h^{\prime} \leqslant h$ and $k^{\prime} \leqslant k$. As we have already seen, since $z_{\left[h^{\prime}, k^{\prime}\right]}=z_{[h, k]}$, this would imply that $\left(h^{\prime}, k^{\prime}\right)=(h, k)$. Since $h^{\prime} \leq i \leq h$ and $k^{\prime} \leq j \leq k$, we would finally have $(h, k)=(i, j)$, in contradiction with our hypotheses.

Finally, we have

$$
\frac{\partial}{\partial z_{[i, j]}} \operatorname{det} M_{z 0, \#(i, j)}^{*}\left(z_{0}\right)= \pm \operatorname{det} M_{z_{0}}^{*}\left(z_{0}\right)+\sum_{\left(i^{\prime}, j^{\prime}\right)} \pm \operatorname{det} N_{i^{\prime}, j^{\prime}}\left(z_{0}\right)
$$

where the sum is taken on all pairs $\left(i^{\prime}, j^{\prime}\right) \in\left(\mathcal{R}\left(M_{z_{0}, \#(i, j)}^{*}\right) \times \mathcal{C}\left(M_{z_{0}, \#(i, j)}^{*}\right)\right)$ such that $z_{\left[i^{\prime}, j^{\prime}\right]}=z_{[i, j]}$. We conclude as above that all the terms in the sum vanish.

\subsection{The analytic case}

We prove Theorem 3.3 by recurrence on $p$. When $p=0$, we have $A=A_{1}$ and hence the statement follows from Theorem 3.2.

Since $A_{0}$ is defined by analytic conditions (at least in a suitable chart), it is a stratified set. It suffices to bound the codimension of the stratum $S \subset A_{0}$ of maximal dimension. Let us consider the restricted projection

$$
\left(\pi_{p-1 \mid S}^{p}\right): S \rightarrow J^{p-1}(X, Y) .
$$

and the associated rank map

$$
S \ni a \mapsto \operatorname{rank} d_{a}\left(\pi_{p-1_{\mid S}}^{p}\right) \in\left\{0,1, \ldots, \min \left\{\operatorname{dim} S, \operatorname{dim} J^{p-1}(X, Y)\right\}\right\} .
$$

Let us also consider an open subset $U$ of $S$ such that the rank map is constant on $U$. Such a subset exists: for instance we can take as $U$ the preimage of the maximum 
value attained by the map (this preimage is open because the rank map is lowersemicontinuous).

It follows from the constant-rank theorem that, up to further restricting $U$ if necessary, $\pi_{p-1}^{p}(U)$ is a submanifold of $J^{p-1}(X, Y)$. Let us call $V$ this manifold. We claim that

$$
U \subseteq \tilde{V}
$$

where $\tilde{V} \subseteq J^{p}(X, Y)$ is defined according to (3.1), i.e.

$$
\tilde{V}=\left\{j_{x}^{p} f \in J^{p}(X, Y): j^{p-1} f \text { is not transverse to } V \text { at } x\right\} .
$$

Since the conclusion of Theorem 3.3 is assumed to be true for $p-1$, we have $\operatorname{codim} \tilde{V} \geq n+1$, hence the claim implies $\operatorname{codim} U \geq n+1$. Since $U$ is open in $S$ and $S$ is the stratum of maximal dimension, we get

$$
\operatorname{codim} A_{0}=\operatorname{codim} S=\operatorname{codim} U \geq n+1,
$$

which proves the proposition. Let us now prove the claim $U \subseteq \tilde{V}$. Given any $a=j_{x}^{p} f \in U$, we have

$$
T_{\pi_{p-1}^{p} a} V=\left(\pi_{p-1 \mid S}^{p}\right)\left(T_{a} U\right) \subseteq \pi_{p-1}^{p}\left(T_{a} A\right) .
$$

Here the first equality follows by the constant-rank theorem, while the inclusion follows from the fact that $U \subseteq S \subseteq A$. Moreover, the very definition of $A_{0}$ yields

$$
\pi_{p-1}^{p}\left(T_{a} A\right)+E^{p-1}(a) \subsetneq T_{\pi_{p-1}^{p} a} J^{p-1}(X, Y) .
$$

It follows that

$$
T_{\pi_{p-1}^{p} a} V+E^{p-1}(a) \subseteq \pi_{p-1}^{p}\left(T_{a} A\right)+E^{p-1}(a) \subsetneq T_{\pi_{p-1}^{p} a} J^{p-1}(X, Y)
$$

which implies that $a \in \tilde{V}$, as desired.

\section{References}

[1] R. Abraham and J. Robbin, “Transversal Mappings and Flows”, Benjamin, 1967.

[2] N. ARONSZAJN, Differentiability of Lipschitzian mappings between Banach spaces, Studia Math. 57 (1976), 147-190.

[3] Y. Benyamini and J. Lindenstrauss, "Geometrical Nonlinear Functional Analysis", Vol. 1, AMS Colloquium Publications, Vol. 48, 2000.

[4] P. BERNARD, On the number of Mather measures of Lagrangian systems, Arch. Ration. Mech. Anal. 197 (2010), 1011-1031.

[5] M. Gromov, "Partial Differential Relations", Springer, 1986.

[6] J. P. R. Christensen, On sets of Haar measure zero in abelian Polish groups, Israel J. Math. 13 (1972), 255-260. 
[7] M. CsÖRnYEI, Aronszajn null and Gaussian null sets coincide, Israel J. Math. 111 (1999), 191-201.

[8] Y. Eliashberg and N. MishacheV, "Introduction to the $h$-principle", Graduate Studies in Math., Vol. 48, AMS, 2002.

[9] M. W. HIRSCH, "Differential Topology", Springer, 1976.

[10] B. R. Hunt and V. Kaloshin, Prevalence, In: "Handbook of Dynamical Systems", Vol. 3, Elsevier, 2010, 73-87.

[11] B.R HUnT, T. SAUER RM AND J.A. YORKE, Prevalence: a translation-invariant "almost every” on infinite-dimensional spaces, Bull. Amer. Math. Soc. (N.S.) 27 (1992), 217-238; with an addendum in Bull. Amer. Math. Soc. (N.S.) 28 (1993), 306-307.

[12] M. Golubitsk Y and V. Guillemin, "Stable Mappings and their Singularities", Springer, 1973.

[13] R. JOLY, Adaptation of the generic PDE's results to the notion of prevalence, J. Dyn. Differential Equations 19 (2007), 967-983.

[14] L. ZAJÍČEK, On Lipschitz and d.c. surfaces of finite codimension in a Banach space, Czech. Math. J. 58 (2008), 849-864.

Université Paris-Dauphine

CEREMADE, UMR CNRS 7534

Pl. du Maréchal de Lattre de Tassigny 75775 Paris Cedex 16, France patrick.bernard@ceremade.dauphine.fr mandorino@ceremade.dauphine.fr 